\title{
Efficacy and safety of golimumab as add-on therapy to standard disease-modifying antirheumatic drugs: Results of the GO-MORE study in the Polish population
}

\author{
Sławomir Jeka ${ }^{1, A, B, D-F}$, Bogdan Batko ${ }^{2, B-F}$, Mariusz Korkosz ${ }^{3, B-F}$, Maria Majdan ${ }^{4, B-F}$, Brygida Kwiatkowska ${ }^{5, B-F}$, \\ Iwona Dankiewicz-Fares ${ }^{1, B-F}$, Jerzy M. Sobiecki ${ }^{6,-F}$, Włodzimierz Samborski ${ }^{7, B-F}$ \\ 1 Department of Rheumatology and Connective Tissue Diseases, The Jan Biziel University Hospital No. 2, Nicolaus Copernicus University in Torun, Collegium Medicum in Bydgoszzz, Poland \\ 2 Department of Rheumatology, The Józef Dietl Specialist Hospital, Kraków, Poland \\ ${ }^{3}$ Division of Rheumatology, Department of Internal Medicine and Gerontology, Jagiellonian University Medical College, Kraków, Poland \\ ${ }^{4}$ Department of Rheumatology and Connective Tissue Diseases, Medical University of Lublin, Poland \\ ${ }^{5}$ Department of Early Arthritis, National Institute of Geriatrics, Rheumatology and Rehabilitation, Warszawa, Poland \\ ${ }^{6}$ Department of Medical Affairs, Merck Sharp \& Dohme, Warszawa, Poland \\ ${ }^{7}$ The Wiktor Dega Orthopedic-Rehabilitative Clinical Hospital, Poznań, Poland
}

A - research concept and design; $\mathrm{B}$ - collection and/or assembly of data; $\mathrm{C}$ - data analysis and interpretation;

$\mathrm{D}$ - writing the article; $\mathrm{E}$ - critical revision of the article; $\mathrm{F}$ - final approval of the article

Address for correspondence

Sławomir Jeka

E-mail: s.jeka@wp.pl

\section{Funding sources}

Sławomir Jeka, Bogdan Batko, Mariusz Korkosz, Maria Majdan, Brygida Kwiatkowska, and Włodzimierz Samborski, and Bogdan Batko have received honoraria as advisory board members and speakers from MSD Polska Sp. z 0.0. Jerzy M. Sobiecki is a permanent employee of MSD Polska Sp. Z 0.0 .

Conflict of interest

Iwona Dankiewicz-Fares declares no conflict of interest.

\section{Acknowledgements}

The authors would like to thank Proper Medical Writing Sp. z 0.0. for language and technical corrections of the manuscript, which were sponsored by MSD Polska Sp. z 0.0 . We also thank the following study investigators who enrolled patients into the study: Jarosław Marcinkiewicz (Sopot, Poland), Jerzy Supronik (Białystok, Poland), RobertZwolak (Lublin, Poland), Maria RellBakalarska (Warszawa, Poland), Anna Dudek (Warszawa, Poland)

Received on July 19, 2016

Reviewed on October 1, 2016

Accepted on January 31, 2017

\section{Abstract}

Background. The GO-MORE study was an open-label, multinational, prospective study that investigated the efficacy and safety of adding golimumab to synthetic disease-modifying antirheumatic drugs (sDMARDs) in patients with active rheumatoid arthritis (RA).

Objectives. The aim of this study was to assess the efficacy and safety of golimumab add-on therapy in the Polish subpopulation of the GO-MORE study.

Material and methods. Patients were administered $50 \mathrm{mg}$ subcutaneous doses of golimumab once a month for 6 months, while continuing therapy with sDMARDs and/or glucocorticoids (GCS). The primary clinical endpoint was the proportion of patients with moderate or good European League Against Rheumatism (EULAR) response based on the 28-joint disease activity score (DAS28) erythrocyte sedimentation rate (ESR) after 6 months.

Results. The Polish subpopulation (129 patients) was similar to the overall study population (3,280 patients) with regard to age, sex, mean baseline DAS28, inflammatory markers, average methotrexate dose, and GCS use; however, they had a longer disease duration (median: 6.04 vs 4.9 years) and more Polish patients (85.9\% vs 78.7\%) had high disease activity (DAS28-ESR $\geq 3.2$ ). At 6 months, $84.5 \%$ of Polish patients showed good or moderate EULAR response, $26.4 \%$ had low disease activity and $17.1 \%$ were in clinical remission, compared with $82.9 \%, 37.4 \%$ and $23.9 \%$, respectively, in the overall study population. Golimumab safety profile was consistent with previous studies and comparable to the overall study population.

Conclusions. The addition of golimumab to sDMARD therapy in Polish RA patients showed good or moderate EULAR DAS28-ESR response in $84.5 \%$ of patients, mirroring the overall study population.

Key words: methotrexate, rheumatoid arthritis, golimumab, disease-modifying antirheumatic drugs

DOI

$10.17219 /$ acem $/ 68737$

Copyright

Copyright by Author(s)

This is an article distributed under the terms of the Creative Commons Attribution Non-Commercial License (http://creativecommons.org/licenses/by-nc-nd/4.0/) 


\section{Introduction}

According to the current recommendations for the management of rheumatoid arthritis (RA), the main treatment goal is to attain remission of the disease symptoms, or alternatively to achieve low disease activity in patients who fail to attain remission with the available treatment methods. ${ }^{1-3}$ The recommendations specify time periods in which this treatment goal should be achieved; i.e., improvement should be achieved within 3 months and the treatment goal (remission or low disease activity) should be met within 6 months. ${ }^{1,2}$ To achieve this goal, synthetic disease-modifying antirheumatic drugs (sDMARDs) are recommended in the $1^{\text {st }}$ phase of RA treatment. The first-line conventional sDMARD is methotrexate (MTX). If MTX is not tolerated or contraindicated, then leflunomide, sulfasalazine or hydroxychloroquine, alone or in combination with glucocorticoids (GCS), are recommended. In case of inefficacy or adverse reactions, other conventional sDMARDs or biologic agents are recommended. The recommended first-line biologics include tumor necrosis factor alpha (TNF- $\alpha)$ inhibitors, abatacept, tocilizumab, and, in certain conditions, rituximab. $^{2}$

Table 1. Baseline clinical and demographic characteristics of the GO-MORE study patients, including the Polish population ${ }^{9}$

\begin{tabular}{|c|c|c|c|}
\hline \multicolumn{2}{|c|}{ Characteristics } & Overall study population & Polish patient group \\
\hline \multicolumn{2}{|c|}{ Number of patients in the study } & 3280 & 129 \\
\hline \multirow{2}{*}{ Age [years] } & mean (SD) & $52.3(12.8)$ & $49.7(11.06)$ \\
\hline & median (min, max) & $53.0(18,88)$ & $52.0(18,78)$ \\
\hline \multicolumn{2}{|l|}{ Females, n (\%) } & $2716(86.8)$ & $111(86)$ \\
\hline \multirow{2}{*}{ Race, n (\%) } & Caucasian & $2283(69.6)$ & $129(100)$ \\
\hline & other & $997(30.4)$ & 0 \\
\hline \multicolumn{2}{|c|}{ BMI [kg/m²], median (min, max) } & $26.2(14.0,54.5)$ & $24.96(16.7,42.6)$ \\
\hline \multirow{2}{*}{ Disease duration [years] } & mean (SD) & $7.6(7.9)$ & $8.78(8.69)$ \\
\hline & median (min, max) & $4.9(0.01,56.6)$ & $6.04(0.12,44.6)$ \\
\hline \multirow{3}{*}{ DAS28-ESR } & n & 3270 & 129 \\
\hline & mean (SD) & $5.97(1.1)$ & $5.96(0.94)$ \\
\hline & high disease activity (>5.1), n (\%) & $2572(78.7)$ & $110(86)$ \\
\hline DAS28-CRP & mean (SD) & $5.41(1)$ & $5.34(0.9)$ \\
\hline $\mathrm{CRP}[\mathrm{mg} / \mathrm{L}]$ & mean (SD) & $14.48(20.38)$ & $13.46(18.62)$ \\
\hline $\mathrm{ESR}[\mathrm{mm} / \mathrm{h}]$ & mean (SD) & $34.9(24.64)$ & $32.8(18.98)$ \\
\hline \multirow{2}{*}{ Anti-CCP } & $n$ & 3225 & 129 \\
\hline & positive ( $\geq 20 \mathrm{U} / \mathrm{mL}), \mathrm{n}(\%)$ & $2318(71.9)$ & $102(79)$ \\
\hline \multirow{2}{*}{ Rheumatoid factor } & $n$ & 3234 & 129 \\
\hline & positive ( $\geq 15 \mathrm{U} / \mathrm{mL}), \mathrm{n}(\%)$ & $2344(72.5)$ & $127(98)$ \\
\hline \multirow{4}{*}{ HAQ-DI } & mean (SD) & $1.44(0.67)$ & $1.51(0.57)$ \\
\hline & $n$ & 3270 & 129 \\
\hline & methotrexate monotherapy, n (\%) & $1681(51.4)$ & $87(67)$ \\
\hline & methotrexate + leflunomide, n (\%) & $216(6.6)$ & $1(1)$ \\
\hline \multirow{4}{*}{ sDMARD } & methotrexate + sulfasalazine, n (\%) & $150(4.6)$ & $8(6)$ \\
\hline & methotrexate + hydroxychloroquine, chloroquine, n (\%) & $433(13.2)$ & $10(8)$ \\
\hline & $\begin{array}{l}\text { methotrexate }+ \text { hydroxychloroquine, chloroquine + } \\
\text { sulfasalazine, n (\%) }\end{array}$ & $106(3.2)$ & $1(1)$ \\
\hline & leflunomide monotherapy, n (\%) & $303(9.3)$ & $8(6)$ \\
\hline \multirow{2}{*}{ Glucocorticoids } & $n$ & 3280 & 129 \\
\hline & GCS treatment $(\%)$ & $2078(63.4)$ & $85(66)$ \\
\hline \multirow{4}{*}{$\begin{array}{l}\text { Number of previously } \\
\text { failed sDMARDs }\end{array}$} & $n$ & 3279 & 129 \\
\hline & 1, n (\%) & 1129 (34.4) & $28(22)$ \\
\hline & $2, n(\%)$ & 1176 (35.9) & $48(37)$ \\
\hline & $\geq 3, \mathrm{n}(\%)$ & $974(29.7)$ & $53(41)$ \\
\hline
\end{tabular}

anti-CCP - anti-cyclic citrullinated peptide antibody; BMI - body mass index; CRP - C-reactive protein; DAS28 - 28-joint disease activity score; DMARD - disease-modifying antirheumatic drugs; ESR - erythrocyte sedimentation rate; GCS - glucocorticoids; HAQ-DI - health assessment questionnaire disability index. 
A new generation of TNF- $\alpha$ inhibitor is golimumab, an anti-TNF- $\alpha$ monoclonal antibody that is administered subcutaneously (SC) once a month. Golimumab has been extensively evaluated in placebo-controlled RA clinical studies; it has shown efficacy in MTX-naïve patients, in patients with inadequate response to MTX, in patients previously treated with at least one TNF- $\alpha$ inhibitor. Furthermore, golimumab can inhibit radiographic progression in the joints of MTX-naive patients and of those with inadequate MTX response. ${ }^{4-8}$ GO-MORE study was the first to evaluate the efficacy and safety of golimumab as an add-on therapy to different sDMARDs and to low doses (<15 mg/week) of MTX. ${ }^{9}$

The GO-MORE study evaluated golimumab as an addon therapy (alone or in combination) to MTX, leflunomide, sulfasalazine, hydroxychloroquine, and chloroquine. This mimics the real-life clinical situation, when golimumab is typically administered in conjunction with different DMARDs.

The GO-MORE study included 129 Caucasian Polish patients from 13 centers across Poland. As the response to and safety of synthetic and biological DMARDs including golimumab may be genetically determined, and could be affected by different standards of treatment in various countries, the results of the GO-MORE study may vary across populations. ${ }^{10,11}$ Therefore, we set out to investigate whether the efficacy of golimumab in a Polish subpopulation differed from the international study population, which combined ethnic groups and standards of treatment.

\section{Objectives}

The objective of the paper was to evaluate the efficacy and safety of golimumab as an add-on therapy to standard DMARDs in the Polish patient subgroup (129 patients) from the $1^{\text {st }}$ part of the GO-MORE study, and to compare the efficacy results with worldwide study population.

\section{Material and methods}

This was a post-hoc analysis of the GO-MORE study (P06129; NCT00975130) which evaluated the efficacy and safety of golimumab in RA patients under conditions closely resembling routine clinical practice. ${ }^{9}$ The GOMORE study was an open-label, prospective, multicenter clinical trial that was conducted in 40 countries across Europe, Asia, North America, South America, and Africa, and involved 475 centers. It was approved by Bioethics Committees and conducted according to Good Clinical Practice and Declaration of Helsinki.

The study enrolled 3,280 patients with RA, aged over 18 years, diagnosed according to the revised 1987 criteria of the American College of Rheumatology. ${ }^{12}$ Disease activity was evaluated using the 28-joint disease activity score (DAS28). The study patients had active form of the disease (DAS28-ESR $\geq 3.2$ ) despite treatment with 1 or more of the following sDMARDs at stable doses for at least 1 month: MTX, sulfasalazine, hydroxychloroquine, chloroquine, chloroquine phosphate, leflunomide, gold salts, azathioprine, or cyclosporine. The exclusion criterion was prior use of biologics and any contraindications for TNF- $\alpha$ inhibitor use.

The study consisted of 2 parts. In part 1 , the patients received $50 \mathrm{mg}$ of SC golimumab, once a month for 6 months. Throughout the study the patients continued taking their regular doses of sDMARDs and/or GCS. Part 2 of the study included patients who did not attain remission after 6 months, but achieved good or moderate response according to the EULAR criteria. ${ }^{13}$ The patients were randomly assigned (1:1) to one of 2 treatment groups receiving for the following 6 months $50 \mathrm{mg}$ of SC golimumab once a month or combination regimen of SC and IV golimumab. Patients who did not participate in the study part 2 could continue golimumab therapy until week 48 as an extension of part 1 .

The primary endpoint of part 1 was the percentage of patients who achieved good or moderate EULAR response (defined as DAS28-ESR improvement of $>1.2$ from a baseline score or an improvement of $0.6-1.2$ in the case of a baseline score of $\leq 5.1$ ). The key secondary endpoints included percentage of remissions and low disease activity according to DAS28-ESR, DAS28 calculated with C-reactive protein (CRP), simplified disease activity index (SDAI) and percentage of patients who developed minimal or no functional impairment (health assessment questionnaire disability index, HAQ-DI, $\leq 0.5$ ). The essential efficacy evaluation criterion for both therapeutic regimens in part 2 was the percentage of patients who attained DAS28-ESR remission at the start of month 11 and at the end of month 12. Part 2 of the study included 505 patients with only a small number of Polish subjects (13), and therefore, this paper presents an analysis of results obtained with the Polish population participating in part 1 of the study.

\section{Results}

\section{Baseline characteristics of the Polish patient group}

The overall results of the GO-MORE study have been previously reported. ${ }^{9}$

The Polish GO-MORE clinical trial included 129 patients (Table 1). The majority (83\%) of the patients enrolled in the study used MTX, and most took high doses of MTX ( $\geq 15 \mathrm{mg} /$ week). A significantly lower proportion of patients (33\%) took a sDMARD other than MTX, and only $16 \%$ of these patients received a SDMARD in combination with 
MTX. The second most frequently used sDMARD was leflunomide ( $6 \%$ of patients in the Polish subpopulation and $9.3 \%$ of patients in the overall study population), and the remaining patients received a combination treatment, mostly MTX with chloroquine or hydroxychloroquine (13\%). Tritherapy (MTX, hydroxychloroquine/chloroquine and sulfasalazine) was administered to only $1 \%$ of patients in the Polish subpopulation compared to $3.2 \%$ of patients in the overall population. The baseline characteristics of the Polish group of patients were similar to the overall study population in terms of age, sex, the mean baseline DAS28, HAQ-DI value, number of painful and swollen joints, laboratory inflammation markers, MTX dose, and percentage of patients treated with GCS (Table 1). Compared to the overall study population, the Polish patient population was characterized by a longer disease duration (median: 6.04 vs 4.9 years), a higher percentage of patients with high disease activity according to DAS28-ESR (86\% vs $78.7 \%$ of patients), and a higher number of previous failures with at least 3 sDMARDs ( $41 \%$ vs $29.7 \%$ of patients).

\section{Efficacy of golimumab}

After 6 months of golimumab treatment, 85\% (109/129) of the patients in the Polish subpopulation achieved good or moderate EULAR response, which was the primary part 1 endpoint. Low disease activity (DAS28-ESR < 3.2) after 6 months of treatment was achieved by $26 \%(34 / 129)$ and remission (DAS28-ESR < 2.6) was achieved by $17 \%$ $(22 / 129)$ of patients. The percentage of patients who met the criterion of good or moderate EULAR response after 6 months (85\%) was similar to the overall study population mean of $82.1 \%$. The percentage of low disease activity and remission achieved after 6 months in the Polish patient population was lower than in the overall study population, i.e., $26 \%$ vs $37.4 \%$ and $17 \%$ vs $23.9 \%$, respectively (Table 2 ).

The percentage of Polish patients who met the criteria of good or moderate EULAR response increased over time (i.e., $58 \%$ at the beginning of month $2,78 \%$ at the beginning of month 4 , and $85 \%$ at the end of month 6), which was similar to the overall population data (i.e., $64.9 \%, 76.9 \%$ and $82.1 \%$, respectively). The percentage of Polish patients who attained remission over time also increased (i.e., to $3 \%$, $12 \%$ and $17 \%$, respectively), but these percentages were lower than in the overall population (i.e., 7.7\%, 16.1\% and $17.1 \%$, respectively) (Table 2). There was a mean decrease in DAS28-ESR within 6 months of golimumab therapy from 5.96 (baseline) to $2.19( \pm 1.19)$.

The mean baseline SDAI score decreased from 35.16 at baseline to $21.96( \pm 12.23)$ at the end of treatment month 6. A total of $45 \%$ of patients (58/129) achieved low disease activity (SDAI < 11) and $10 \%$ of patients $(13 / 129)$ achieved remission (SDAI < 3.3) after 6 months. Similar to the results seen with DAS28-ESR, the percentage of Polish patients achieving low disease activity according to SDAI increased over time, reaching $16 \%, 32 \%$ and $45 \%$ in months 2, 4 and 6, respectively. The percentage of Polish patients achieving remission according to SDAI also increased over time to $2 \%, 8 \%$ and $10 \%$ in months 2,4 and 6 , respectively. In the Polish group, these percentages based

Table 2. Percentage of patients who achieved good or moderate response, low disease activity or remission, according to DAS28-ESR (EULAR criteria) at subsequent measurement points. Results obtained in the Polish cohort $(n=129)$ and in the overall study population $(n=3280)$ are presented for comparison ${ }^{9}$

\begin{tabular}{|c|c|c|c|c|c|c|}
\hline \multirow[b]{2}{*}{ DAS28-ESR (EULAR) } & \multicolumn{2}{|c|}{ Beginning of month 2} & \multicolumn{2}{|c|}{ Beginning of month 4} & \multicolumn{2}{|c|}{ End of month 6} \\
\hline & $\begin{array}{c}\text { Overall } \\
\text { population }\end{array}$ & $\begin{array}{l}\text { Polish patient } \\
\text { group }\end{array}$ & $\begin{array}{c}\text { Overall } \\
\text { population }\end{array}$ & $\begin{array}{l}\text { Polish patient } \\
\text { group }\end{array}$ & $\begin{array}{c}\text { Overall } \\
\text { population }\end{array}$ & $\begin{array}{l}\text { Polish patient } \\
\text { group }\end{array}$ \\
\hline $\begin{array}{l}\text { Patients who achieved low disease activity } \\
(\text { DAS28-ESR < 3.2) }(\%)\end{array}$ & 16.6 & 9 & 28.1 & 21 & 37.4 & 26 \\
\hline $\begin{array}{l}\text { Patients who achieved remission } \\
(\text { DAS28-ESR < 2.6) }(\%)\end{array}$ & 7.7 & 3 & 16.1 & 12 & 24 & 17 \\
\hline
\end{tabular}

Table 3. Evaluation of Polish patients' functional improvement during golimumab treatment $(n=129)$

\begin{tabular}{|l|c|c|c|}
\hline & $\begin{array}{c}\text { Before treatment } \\
\text { (baseline) }\end{array}$ & $\begin{array}{c}\text { Beginning } \\
\text { of month } 2\end{array}$ & $\begin{array}{c}\text { Beginning } \\
\text { of month } 4\end{array}$ \\
\hline No or minimal functional impairment(HAQ-DI $\leq 0.5), n(\%)$ & $8(6)$ & $18(14)$ & $26(20)$ \\
\hline Minimal (acceptable) disease symptoms(PASS), $n(\%)$ & $14(11)$ & $59(46)$ & $25(19)$ \\
\hline
\end{tabular}

HAQ-DI - health assessment questionnaire disability index; PASS - patient acceptable symptom state.

Table 4. The effect of golimumab on Polish patients' quality of life as determined by the EQ-5D $(n=129)$

\begin{tabular}{|c|c|c|c|c|}
\hline & Before treatment (baseline) & Beginning of month 2 & Beginning of month 4 & End of month 6 \\
\hline EQ-5D (mean \pm SD) & $0.44 \pm 0.28$ & $0.15 \pm 0.22$ & $0.19 \pm 0.26$ \\
\hline
\end{tabular}


on SDAI were lower than the percentages of low disease activity and remission determined by the DAS28-ESR in the overall study population.

\section{Subgroup analysis of efficacy of golimumab}

A subgroup analysis of the Polish patients showed no significant differences in the percentages of patients who achieved good or moderate EULAR response at the end of month 6 due to the dose of MTX, the use of DMARDs other than MTX, the number of previously failed DMARDs, and the use or non-use of GCS.

\section{Effect of golimumab on quality of life}

The effect of golimumab treatment on the patients' physical function was measured in all patients using the HAQDI score. Prior to the treatment, the patients' general physical function measured by HAQ-DI was $1.51 \pm 0.568$, and disease activity evaluation by the patient using visual analogue scale (VAS) 0-100 mm was $63.56 \pm 17.357$. Golimumab treatment significantly improved the patients' functional condition and more patients reported symptoms that were acceptable and did not disturb their everyday life (Table 3). In addition to the functional condition improvement, the patients treated with golimumab also reported an improved quality of life, as measured by the EQ-5D questionnaire (Table 4). The percentage of Polish patients without a disability or with minimal impairment of function (HAQ-DI $\leq 05$ ) increased from $6 \%$ at baseline to $14 \%$, $20 \%$ and $19 \%$ at the beginning of month 2, 4 and the end of month 6, respectively. In addition, the percentage of Polish patients exhibiting minimal (acceptable) disease symptoms (patient acceptable symptom state, PASS) increased from $11 \%$ at baseline to $46 \%, 57 \%$ and $73 \%$ at the beginning of month 2, 4 and the end of month 6, respectively. Therefore, treatment with golimumab significantly improved patients' physical function as assessed by HAQ-DI, and significantly improved health-related quality of life.

\section{Safety and tolerability of golimumab}

Golimumab safety was evaluated in all patients in the study in relation to adverse events observed during treatment (treatment emergent adverse event, TEAE). The percentage of Polish patients with at least 1 TEAE was $45 \%$ in part 1 of the study, and $15 \%$ of patients had a drugrelated TEAE (Tables 5, 6). Abnormal laboratory results were observed in $3 \%$ of the patients, with the most common being elevated serum aminotransferase, elevated potassium, decreased thyroid stimulating hormone, or decreased fibrinogen. No deaths occurred during part 1 of the study or within 30 days after the last dose. Therefore, golimumab was well-tolerated in the Polish cohort, and the safety profile was consistent with previous studies on the drug.

\section{Discussion}

In this analysis of the GO-MORE study in the Polish subpopulation (129 patients), we found that SC treatment with $50 \mathrm{mg}$ of golimumab once a month for 6 months, in combination with different sDMARDs, resulted in good or moderate EULAR response in the majority of patients (85\%). By the $2^{\text {nd }}$ month of treatment, more than half of the Polish patients (58\%) had already achieved good or moderate EULAR response, and after 4 months, this proportion had increased to $78 \%$ of the patients. The data is similar to that observed in the overall GO-MORE study population. ${ }^{9}$ On the other hand, remission rates in the Polish subpopulation improved more gradually; $3 \%, 12 \%$ and $17 \%$ of patients had achieved remission at the beginning

Table 5. Adverse events in the Polish patient subpopulation $(n=129)$ during golimumab treatment

\begin{tabular}{|l|c|}
\hline \multicolumn{1}{|c|}{ Categories of adverse events } & $\mathrm{n}(\%)$ \\
\hline All adverse events & $59(45)$ \\
\hline Drug-related adverse events & $20(15)$ \\
\hline Serious adverse events & $4(3)$ \\
\hline Adverse events leading to early withdrawal & $3(2)$ \\
\hline Deaths & 0 \\
\hline Adverse injection site reactions & 0 \\
\hline Clinically significant abnormal laboratory results & $5(3)$ \\
\hline
\end{tabular}

Table 6. Adverse events in the Polish patient subpopulation $(n=129)$ during 6 months of golimumab treatment according to various organ system manifestations

\begin{tabular}{|l|c|}
\hline \multicolumn{1}{|c|}{ Organ system manifestations } & $\begin{array}{c}\text { Adverse events, } \\
n(\%)\end{array}$ \\
\hline Infections & $31(24)$ \\
\hline Musculoskeletal system & $8(6)$ \\
\hline Gastrointestinal system & $7(5)$ \\
\hline Respiratory system & $6(5)$ \\
\hline Skin & $6(5)$ \\
\hline General symptoms & $5(4)$ \\
\hline Abnormal laboratory results & $5(3)$ \\
\hline Metabolic disorders & $4(3)$ \\
\hline Arterial hypertension & $4(3)$ \\
\hline Hematologic disorders & $3(2)$ \\
\hline Neoplasms: benign, malignant and unspecified & $3(2)$ \\
\hline Nervous system & $3(2)$ \\
\hline Dysrhythmias & $2(2)$ \\
\hline & $1(1)$ \\
\hline Discopathy & $1(1)$ \\
\hline Skin melanoma & $1(1)$ \\
\hline Dysrhythmias & events (SAE), n (\%) \\
\hline Endometrial hyperplasia & $1(1)$ \\
\hline
\end{tabular}


of month 2, 4 and the end of month 6, respectively. Therefore, although the clinical response with a combined golimumab therapy is achieved relatively early, it may take more time to achieve the main RA treatment goals, such as remission or low disease activity.

The remission rate (17\%) observed in the Polish subpopulation using golimumab is similar to that observed previously using other anti-TNF- $\alpha$ drugs. For example, Hyrich et al. found that the remission rates (measured by DAS28) for 4000 RA patients, monitored after the $1^{\text {st }}$ year, and subsequently from 2001 to 2008, after treatment with different anti-TNF $\alpha$ drugs (i.e., etanercept, infliximab and adalimumab) ranged from $8 \%$ to $19.4 \% .{ }^{10}$ On the other hand, in the CORRONA study, remission rates measured by DAS28 were slightly higher; after 6 months of adalimumab (874 patients), etanercept (640 patients) or infliximab (728 patients) therapy, remission rates were $25.2 \%$, $28.4 \%$ and $28.2 \%$ of patients, respectively. ${ }^{14}$ However, this difference in remission rates may have occurred as the patients in the CORRONA study had lower mean baseline disease activity (DAS28 $=4.4$ ) compared to the Polish subpopulation of the GO-MORE study (DAS28 = 5.96). Moreover, the remission rate was lower after 6 months of treatment in the Polish subpopulation (17\%) compared to the overall GO-MORE study population (23.9\%). This difference may be due to the fact that the Polish patients had a longer disease duration compared to the overall study population (6.04 vs 4.9 years) and, therefore, they were most likely to be in a more advanced stage of the disease. Indeed, there were more Polish patients with high disease activity (i.e., DAS28-ESR > 5.1) compared to the overall study population ( $86 \%$ vs $78.7 \%$ ). There was also a larger proportion of Polish patients with the presence of rheumatoid factor and/or anti-cyclic citrullinated peptide compared to the overall study population (99\% vs $72.5 \%$ and $79 \%$ vs $71.9 \%$, respectively), which usually indicates poorer outcomes in RA patients. In addition, the Polish subpopulation had a higher proportion of patients who had been previously unsuccessfully treated with at least 3 sDMARDs compared to the overall study population (41\% vs $29.7 \%$ ). Finally, there are differences in access to drugs and in treatment standards between Poland and other countries examined in the GO-MORE study, which could explain the minor differences in remission rates to golimumab treatment in the Polish subpopulation compared to the entire study population.

We also found that the proportion of Polish patients who achieved remission or had low disease activity was lower when measured using SDAI compared to DAS28ESR. Using SDAI, only $10 \%$ of patients achieved remission and $45 \%$ of patients showed low disease activity after 6 months of treatment. This discrepancy between the DAS28 and SDAI results is probably due to the fact that SDAI includes the assessment of the patient's general condition by the physician. The physician may evaluate the patient's symptoms more rigorously than the patient themselves, who have become accustomed to the chronic ailments. Moreover, as the mean DAS28, CRP and ESR values were comparable between the Polish subpopulation and the overall study population, the lower remission and low disease activity rates (according to both DAS28-ESR and SDAI) in Polish patients compared to the overall study population should be interpreted with caution. Indeed, this may simply be due to the fact that the Polish subpopulation had a larger proportion of patients with higher baseline disease activity (DAS28-ESR > 5.1), as mentioned above.

A larger group of patients received MTX in the Polish subpopulation (83\%) compared to the overall study population (79.0\%); $67 \%$ of the Polish subpopulation and 51.4\% in the overall study population were in MTX monotherapy. In addition, a larger proportion of Polish patients received MTX at high doses exceeding $15 \mathrm{mg} /$ week. A significantly lower proportion of patients (33\%) took a sDMARD other than MTX and only $16 \%$ of these patients received a sDMARD in combination with MTX.

The Polish subpopulation of the GO-MORE study showed a significant improvement in terms of physical function and quality of life, and golimumab was effective in patients who had shown insufficient response to previous therapies with 1 or more sDMARDs. The adverse events profile in the Polish subpopulation was similar to the overall study population and to that described in previous studies using golimumab, and was also similar to that observed using other anti-TNF- $\alpha$ drugs. ${ }^{9,11,15,16}$ The most commonly observed adverse events were infections, and serious events were reported in only $3 \%$ of the Polish patients. In addition, no deaths or injection site reactions occurred in the Polish subpopulation.

The basic limitation of this analysis of the Polish subpopulation of the GO-MORE study was the small group of participants (129 patients), which makes it difficult to conduct a statistical analysis of golimumab efficacy among individual subgroups. The subgroup analysis showed no significant differences in the percentages of patients who achieved good or moderate EULAR response at the end of month 6 depending on MTX dose, use of sDMARDs other than MTX, number of failed sDMARDs, and use or non-use of GCS. Despite this, due to the small size of the Polish patient group, and consequently very small subgroups, the data should be interpreted with caution. Nevertheless, taking into account that the overall study population showed no efficacy differences among the abovementioned subgroups, the results obtained in the Polish patients seem to be reliable. ${ }^{9}$ Another potential limitation of part 1 of the GO-MORE study may be its open-label character, which can have an inherent bias. However, results of the observational phase of the study may be most representative for the RA patient population, which is common in rheumatological practice.

In conclusion, the results of the GO-MORE study in the Polish population show the efficacy and safety of golimumab as an add-on treatment to different sDMARDs, 
with or without GCS therapy, in patients with RA in whom previous DMARD therapies had failed, demonstrating good or moderate EULAR DAS28-ESR response in a large proportion of patients (85\%). The onset of action was rapid, with good tolerability, and a safety profile consistent with the data described for golimumab in other clinical trials and in the Summary of Product Characteristics.

\section{References}

1. Smolen JS, Landewe R, Breedveld FC, et al. EULAR recommendations for the management of rheumatoid arthritis with synthetic and biological disease-modifying antirheumatic drugs. Ann Rheum Dis. 2010;69:964-975.

2. Smolen JS, Landewe R, Breedveld FC, et al. EULAR recommendations for the management of rheumatoid arthritis with synthetic and biological disease-modifying antirheumatic drugs: 2013 update. Ann Rheum Dis. 2014;73:492-509.

3. Smolen JS, Aletaha D, Bijlsma WJ, et al. Treating rheumatoid arthritis to target: Recommendations of an international task force. Ann Rheum Dis. 2010;69:631-637.

4. Emery P, Fleischmann RM, Moreland LW, et al. Golimumab, a human antitumor necrosis factor alpha monoclonal antibody, injected subcutaneously every four weeks in methotrexate-naïve patients with active rheumatoid arthritis: Twenty-four week results of a phase III, multicenter, randomized, double-blind, placebo-controlled study of golimumab before methotrexate as first-line therapy for earlyonset rheumatoid arthritis. Arthritis Rheum. 2009;60:2272-2283.

5. Keystone EC, Genovese MC, Klareskog L, et al. Golimumab, a human antibody to tumor necrosis factor given by monthly subcutaneous injections, in active rheumatoid arthritis despite methotrexate therapy: The GO-FORWARD Study. Ann Rheum Dis. 2009;68:789-796.

6. Smolen JS, Kay J, Doyle MK et al.; GO-AFTER study investigators. Golimumab in patients with active rheumatoid arthritis after treatment with tumor necrosis factor alpha inhibitors (GO-AFTER study): A multicentre, randomized, double-blind, placebo controlled, phase III trials. Lancet. 2009;374:210-221.
7. Emery P, Fleischmann R, van der Heijde D, et al. The effects of golimumab on radiographic progression in rheumatoid arthritis. Arthritis Rheum. 2011;63:1200-1210.

8. Keystone EC, Genovese MC, Hall S, et al. Golimumab in patients with active rheumatoid arthritis despite methotrexate therapy: Results through 2 years of the GO-FORWARD study extension. J Rheumatol. 2013;40:1097-1103.

9. Combe B, Dasgupta B, Louw I, et al. Efficacy and safety of golimum$\mathrm{ab}$ as add-on therapy to disease-modifying antirheumatic drugs: Results of the GO-MORE study. Ann Rheum Dis. 2014;73:1477-1486.

10. Hyrich K, Watson $\mathrm{K}$, Lunt $\mathrm{M}$, et al. Changes in disease characteristics and response rates among patients in the United Kingdom starting anti-tumour necrosis factor for therapy for rheumatoid arthritis between 2001 and 2008. Rheumatology (Oxford). 2011;50:117-123.

11. Taylor PC, Ritchin C, Mendelsohn A, et al. Maintenance of efficacy and safety with subcutaneous golimumab among patients with active rheumatoid arthritis who previously received intravenous golimumab. J Rheumatol. 2011;38:2572-2580.

12. Arnett FC, Edworthy SM, Bloch DA, et al. The American Rheumatism Association 1987 revised criteria for the classification of rheumatoid arthritis. Arthritis Rheum. 1988;31:315-324.

13. van Gestel AM, Prevoo ML, van 't Hof MA, et al. Development and validation of the European League Against Rheumatism response criteria for rheumatoid arthritis. Comparison with the preliminary American College of Rheumatology and the World Health Organization/International League Against Rheumatism criteria. Arthritis Rheum. 1996;39:34-40.

14. Greenberg JD, Reed G, Decktor D, et al. A comparative effectiveness study of adalimumab, etanercept and infliximab in biologically naive and switched rheumatoid arthritis patients: Results from the US CORRONA registry. Ann Rheum Dis. 2012;71:1134-1142.

15. Lopez-Olivo MA, Tayar JH, Martinez-Lopez JA, et al. Risk of malignancies in patients with rheumatoid arthritis treated with biologic therapy: A meta-analysis. JAMA. 2012;308:898-908.

16. Galloway JB, Hyrich KL, Mercer LK, et al. Anti-TNF therapy is associated with an increased risk of serious infections in patients with rheumatoid arthritis especially in the first 6 months of treatment: Updated results from the British Society for Rheumatology Biologics Register with special emphasis on risks in the elderly. Rheumatology. 2011;50:124-131. 